\title{
Carbono en biomasa aérea, sistema agroforestal de Theobroma Cacao L., Laboratorio Natural, Los Laureles, 2018
}

\author{
Carbon in aerial biomass, agroforestry system of Theobroma cacao in \\ Los Laureles Natural Laboratory, 2018
}

\author{
Arturo Roberto Avellán Rivera' \\ Enrique Barreto Dolin² \\ Efraín de Jesús Peralta Tercero ${ }^{3}$
}

\section{Resumen}

El estudio se realizó con la finalidad de determinar el carbono almacenado en biomasa aérea del sistema agroforestal (SAF) del laboratorio natural Los Laureles de URACCAN en la comunidad de Hormiguero, municipio de Siuna, en el cual se utilizó el método (no destructivo) mediante aplicación de porcentaje de carbono (50\%) sobre la biomasa seca, sugerido por Intergovernmental Panel on Climate Change, Winrock International, La Organización de Naciones Unidas - Programme on Reducing Emissions from Deforestation and Forest Degradation in Developing Countries. El estudio es cuantitativo, descriptivo y transversal.

El sistema agroforestal es secuencial, con una extensión de 2.84 ha, en el cual se realizó un inventario al 100\% de árboles forestales y frutales; sin embargo, en el cacao se realizó un muestro con una intensidad de $8.45 \%$ distribuyendo 12 parcelas rectangulares de 10 por $20 \mathrm{~m}$ distribuidas sistemáticamente a un distanciamiento de $48.65 \mathrm{~m}$ entre parcela. El Sistema tiene una biomasa aérea de 10.35 toneladas (t), distribuidas de la siguiente manera: árboles frutales, 0.44 t.; especies forestales, $4.70 \mathrm{t}$. y cacao, $5.21 \mathrm{t}$. Es una investigación cuantitativa, descriptiva y transversal.

El sistema agroforestal como banco de almacenamiento de carbono hasta el momento de la recolección de datos tenía 1.44 t carbono por hectárea $(\mathrm{C} / \mathrm{ha}$ ) acumuladas en biomasa aérea de especies forestales y frutales; por otro lado, el componente cacao en su biomasa aérea posee $1.47 \mathrm{t} \mathrm{C} / \mathrm{ha}$, con un total de $2.91 \mathrm{t}$ $\mathrm{C} /$ ha. El valor económico ambiental del almacenamiento de carbono en el laboratorio natural Los Laureles es de $\$ 53.36$ por hectárea.

Palabras clave: frutales; forestales; almacenamiento de carbono; servicio ambiental.

\section{Abstract}

The study was carried out in order to determine the carbon stored in aerial biomass of the agroforestry system (SAF) in Los Laureles Natural Laboratory of URACCAN in Hormiguero community, Siuna municipality, the (non-destructive) method was used by applying carbon percentage $(50 \%)$ on dry biomass, suggested by Intergovernmental Panel on Climate Change, Winrock International, The United Nations - Program on Reducing Emissions from Deforestation and Forest Degradation in Developing Countries. The study is quantitative, descriptive and cross-sectional.

The agroforestry system is sequential, with an extension of 2.84 ha, in which a $100 \%$ inventory of forest and fruit trees was made; however, in the cocoa a sampling with an intensity of $8.45 \%$ was carried

\footnotetext{
1 Ingeniero Agroforestal, No labora, correo: avellanr5@gmail.com ORCID: https://orcid.org/0000-0003-0687-6049

2 Ingeniero Agroforestal, No labora, correo: enriquebarret@icloud.com ORCID: https://orcid.org/0000-0002-4071-4951

3 Master en docencia Universitaria, Ingeniero Forestal, Docente URACCAN, correo: eperaltat11@hotmail.com ORCID: https://orcid.org/0000-0002-5622-3241
} 
out, distributing 12 rectangular plots of 10 by $20 \mathrm{~m}$, systematically distributed at a distance of $48.65 \mathrm{~m}$ between the plot. The System has an aerial biomass of 10.35 tons ( $t$ ), distributed as follows: fruit trees, $0.44 \mathrm{t}$.; forest species, $4.70 \mathrm{t}$. and cocoa, $5.21 \mathrm{t}$. It is a quantitative, descriptive and transversal research.

The agroforestry system as a carbon storage bank until the moment of data collection had $1.44 \mathrm{t}$ carbon per hectare ( $\mathrm{C} / \mathrm{ha}$ ) accumulated in aerial biomass of forest and fruit species; on the other hand, the cocoa component in its aerial biomass has $1.47 \mathrm{t} \mathrm{C} / \mathrm{ha}$, with a total of $2.91 \mathrm{t} \mathrm{C} \mathrm{/} \mathrm{ha.} \mathrm{The} \mathrm{environmental} \mathrm{economic}$ value of carbon storage in Los Laureles Natural Laboratory is $\$ 53.36$ per hectare.

Keywords: fruit trees; forestry; carbon storage; environmental service.

\section{Introducción}

En las regiones cacaoteras de Nicaragua especialmente en las zonas rurales del municipio de Siuna, los productores cultivan este rubro en asociación con otras especies y éstas pueden ser de otros cultivos anuales y/o especies forestales; pero, hay que reconocer que se desconoce el valor potencial que éstos sistemas agroforestales representan como banco de almacenamiento de carbono; los sistemas agroforestales a través del proceso de fotosíntesis capturan el dióxido de carbono atmosférico ( $\left.\mathrm{CO}_{2}\right)$, lo fijan en sus estructuras vivas y parte de éste lo acumulan en su biomasa.

Casanova (2010), realizó un estudio sobre la cuantificación de carbono en sistema agroforestal usando modelos para estimación de carbono en donde afirma, que el potencial de almacenamiento de carbono de los SAF oscila entre 12 a $28 \mathrm{t} \mathrm{C} / \mathrm{ha}-1$, teniendo mayor potencial en las zonas del trópico húmedo y tienen la capacidad de almacenar hasta 70 t $C$ /ha-1 en la vegetación (biomasa aérea) y 25 t/ha-1 en los primeros $20 \mathrm{~cm}$ de profundidad del suelo.

Actualmente el cacao ha tomado mucho más valor y demanda en los mercados nacionales e internacionales, debido a sus variados usos que resultan de éste, por lo cual este rubro ha venido tomando un lugar importante en las tierras productoras donde antes fueron bosques primarios y luego pasaron a ser áreas de cultivos anuales u otros usos.

De acuerdo con el Instituto Nacional forestal (INAFOR, 2016), mediante el programa nacional forestal las tierras forestales de Nicaragua ocupan $34.45 \%$ de la superficie total del territorio nacional; además, el INAFOR (2008), en el inventario nacional forestal asegura que en Nicaragua los bosques han sufrido un desgaste a causa de la deforestación inducida por la agricultura, la ganadería extensiva y los planes de aprovechamientos, perdiendo un promedio anual de 70 mil ha.

Las combinaciones de especies influyen en la conformación de unidades ecológicas con características propias; la conservación de los sistemas agroforestales y su manejo sostenible constituyen una estrategia apropiada de mitigación del cambio climático, al reducir las emisiones de carbono producto de la deforestación y el avance de la frontera agrícola.

Además, el presente estudio aporta de forma positiva al uso, manejo y fortalecimiento de los sistemas agroforestales como una alternativa de cambio desde la realidad local, que involucra sistemas de producción alternativos. También, que sirva como referente de información a productores e instituciones que se perfilan en este campo, así mismo a estudiantes de la universidad, para profundizar en la temática en futura investigaciones.

\section{Revisión de literatura}

Chacón (2007), realizó un inventario sobre almacenamiento de carbono haciendo uso de ecuaciones alométricas sobre la cuantificación de tasas de C; encontró valores de 3.56 t/ha de biomasa en sistemas agroforestales de Talamanca - Costa Rica . Acosta y Tupaz (2007), realizaron investigación sobre captura 
de carbono por la biomasa aérea en dos arreglos agroforestales de la Granja Experimental Botana en el departamento de Nariño- Colombia, mediante modelos alométricos para el cálculo de carbono en árboles; encontrándose con mayor cantidad de biomasa seca aérea total del arreglo cultivo en callejones (CC) con $2.5 \mathrm{t} / \mathrm{ha}$ y carbono almacenado $1.2 \mathrm{t}$ en árboles de A. jorullensis, frente a $0.647 \mathrm{t} / \mathrm{ha}$ de biomasa y de carbono almaceno 0.323 t/ha en el arreglo árboles dispersos (SSP), por lo tanto, el diseño de los arreglos (densidad y/o distancia de siembra) influye sobre la producción de biomasa y el almacenamiento de carbono.

Ortiz, Riascos y Somarriba (2008), realizaron un estudio sobre almacenamiento y fijación de carbono en SAF, encontraron en promedio, un SAF con árbol de laurel acumula $1.86 \mathrm{t}$ de biomasa en condiciones de valle y $3.97 \mathrm{t}$ en loma en 25 años. Un árbol de cacao con diámetro de $30 \mathrm{~cm}$, altura de $5 \mathrm{~m}$, a los 25 años de edad, con incrementos de $1.1 \mathrm{~cm}$ por año; puede acumular, en promedio, $22 \mathrm{~kg}$ de biomasa.

Cerda (2011), realizó una investigación sobre fijación y almacenamiento de carbono en los cacaotales de Valle del cielo Guatemala a los 7 años, a través de modelos para estimación de carbono quien encontró 1.93 t C/ha. Según este autor, en su proyecto "Almacenamiento de carbono en sistemas agroforestales de cacao de Centroamérica”, la mayor acumulación de carbono se da hasta los primeros 10 años de edad.

López (2011), realizó inventario de almacenamiento de carbono en sistemas agroforestales de laurel cacao en Turrialba - Costa Rica con método no destructivo sobre la cuantificación de carbono, en donde estimó que los árboles laurel disperso en plantación de cacao aporta $0.75 \mathrm{t} / \mathrm{ha}$ de carbono al sistema a los 17 años de edad.

Aristizabal y Guerra (2002) en Caldas, Colombia; estimaron el contenido de carbono en un sistema agroforestal laurel- cacao de 15 años, mediante inventarios de carbono usando ecuaciones alométricas recomendadas por ONU-REDD, encontraron valores de $22 \mathrm{t} C$, y una tasa de almacenamiento de $3.05 \mathrm{t}$ $\mathrm{C} /$ ha. En el caso del cacao, aporta $1.93 \mathrm{t} \mathrm{C/ha}$ al sistema, combinados con otras especies y densidad de siembra estable de $4 \mathrm{X}_{3} \mathrm{~m}$.

Estudio realizado por Espinoza, Vásquez y Torres (2012), sobre el almacén de carbono en SAF- café, mediante modelos alométricos, en donde mencionan que los sistemas agroforestales almacenan aproximadamente 9, 21, 50 y 63 t $C$ ha, en la biomasa aérea, raíces y suelo de regiones semiáridas, subhúmedas, húmedas y templadas, respectivamente y el carbono en todos los almacenes se estima entre 12 y 228 t $C$ ha.

En el 2013, Poveda realizó estudio sobre almacenamiento de carbono en sistemas agroforestales de cacao en Waslala, Nicaragua, mediante uso de ecuaciones alométricas para el cálculo de biomasa en los árboles, donde afirma que el almacenamiento de Carbono por componente es de 51.9\% en el suelo, biomasa aérea $35.9 \%$, las raices gruesas con un $7.5 \%$, las raíces delgadas $4.2 \%, 0.3 \%$ para necromasa y hojarasca respectivamente.

La valoración económica de secuestro de carbono se hace mediante la determinación de carbono atrapado por cada componente del sistema agroforestal, donde se determina la tasa promedio de acumulación anual de carbono lo cual se calcula dividiendo los inventarios de $C$ entre la edad del SAF- Cacao. Cerda, (2009) también, hizo una valoración monetaria: primero se multiplica las cantidades de $C$ por un factor de 3.67 para transformarlas a dióxido de carbono $\left(\mathrm{CO}_{2}\right)$ y luego, con base en un precio de $\$ 5$ por $\mathrm{t}$ de $\mathrm{CO}_{2}$, se calculó el valor monetario del almacenamiento y tasas de carbono.

Poveda (2013), realizó un estudio de la valoración económica del servicio ambiental por almacenamiento de carbono en sistemas agroforestales con cacao en el municipio de Waslala, donde determinó un almacenamiento promedio de $91.45 \mathrm{t} \mathrm{C}$ ha, equivalentes a $335.58 \mathrm{t} \mathrm{CO} 2$, el stock actual de carbono estaría valorado \$1.678/ha. El carbono total incremental en los SAF- cacao se estimó en $5.4 \mathrm{t} \mathrm{C} \mathrm{ha/año,}$ equivalentes a $19.8 \mathrm{t} \mathrm{CO} 2 \mathrm{ha} / \mathrm{año}$; por consiguiente, cada productor podría percibir $\$ 99 \mathrm{ha} / \mathrm{año}$. En cuanto a mercados de carbono, pagan solo por el carbono aéreo fijado, en ese caso, la tasa de carbono aéreo fue 2 t $C$ ha/año que equivale a 7.34 t $\mathrm{CO}_{2} / \mathrm{ha}$ y a $\$ 36 \mathrm{ha} / \mathrm{año}$. 


\section{Materiales y métodos}

El estudio se llevó a cabo en el Laboratorio Natural Los Laureles de la Universidad de las Regiones Autónoma de la Costa Caribe Nicaragüense (URACCAN), ubicado en la comunidad El Hormiguero a 20 $\mathrm{Km}$ al noroeste de la ciudad de Siuna, con altura promedio sobre el nivel del mar de $114 \mathrm{~m}$ y coordenadas 708364,1518634. Es Cuantitativo, descriptivo y transversal; cuantitativo puesto que consiste en recolección de datos en campo para probar con base en medición numérica y el análisis estadístico. Descriptivo porque es una sola variable de interés analítico de forma independiente y transversal porque se hizo una sola medición, no cuenta con segunda fase de medición. La muestra fue de $8.45 \%$ de 2.84 ha, dicha muestra es equivalente a 2,400 $\mathrm{m}^{2}$, éste se dividió en 12 parcelas rectangulares de 10 x $20 \mathrm{~m}$ distribuidas sistemáticamente sobre la superficie del sistema agroforestal a un distanciamiento de $48.65 \mathrm{~m}$ entre parcela.

Las variables medidas en campo fueron la determinación del volumen (tocón y fuste). Y el cálculo de las variables carbono por especie y valor económico del carbono almacenado t $C$ ha del SAF. Se realizaron mediciones de diámetros y alturas comerciales de todos los árboles. En caso de los tocones se midieron los diámetros dependiendo de la forma de cada uno (tocones cilíndricos y con gamba). Posteriormente del levantamiento de la información en el campo las muestras extraídas del fuste, se pesó en campo para no tener variaciones en el peso, luego se secó al sol durante 72 horas, después cada 24 horas se realizaron pesajes hasta lograr un peso constante. Los datos fueron depurados y ordenados en el paquete informático de Microsoft Office Excel 2016 para llegar a resultados de las variables en estudio, volumen, densidad básica, biomasa, y carbono de todas las especies encontradas en el SAF.

\section{Resultados y discusión}

En el cuadro 1 se muestra los resultados de volumen total, la biomasa aérea de especies forestales, frutales por hectárea y biomasa total, en donde se cuantificó de mayor contenido de biomasa el cacao, laurel y aguacate canela con $5.21 ; 1.87 ; 1.22$ toneladas respectivamente y las otras especies oscilan de 0.001 a 0.99 toneladas de biomasa aérea.

Cuadro 1. Volumen y biomasa aérea por especie del SAF Los Laureles, 2018.

\begin{tabular}{|l|l|l|l|l|l|l|}
\hline $\mathbf{N}^{\circ}$ & \multicolumn{1}{|c|}{ Especie } & N. árboles total & \multicolumn{1}{|c|}{ Volumen } & \multicolumn{1}{c|}{ B/ha } & \multicolumn{1}{c|}{ Biomasa } & \multicolumn{1}{c|}{$\%$} \\
\hline 1 & Cacao/Theobroma cacao L & 2424 & 208.97 & 1.83 & 5.21 & 50.34 \\
\hline 2 & Aguacate/ Persea americana & 7 & 2.14 & 0.01 & 0.02 & 0.21 \\
\hline 3 & Guaba/ Inga edulis & 61 & 7.42 & 0.12 & 0.35 & 3.41 \\
\hline 4 & Limón/ Citrus aurantifolia & 1 & 0.04 & 0.001 & 0.001 & 0.01 \\
\hline 5 & Mango/ Magnifera indica & 3 & 1.09 & 0.01 & 0.04 & 0.41 \\
\hline 6 & Pijibay/ Bactris gasipaes & 46 & 2.93 & 0.01 & 0.02 & 0.17 \\
\hline 7 & Aguacate Canela/ Persea coerulea & 21 & 23.52 & 0.43 & 1.22 & 11.82 \\
\hline 8 & Cedro Real/ Cedrela Odorata & 8 & 3.96 & 0.04 & 0.12 & 1.14 \\
\hline 9 & Costilla de Danto/ Lecointea amazonica & 2 & 0.34 & 0.01 & 0.03 & 0.26 \\
\hline 10 & Elequeme/ Erythrina poeppigiana & 37 & 8.98 & 0.35 & 0.99 & 9.56 \\
\hline 11 & Guarumo/ Cecropia peltata & 4 & 0.88 & 0.01 & 0.02 & 0.16 \\
\hline 12 & Guácimo molenillo/ Luehea candida & 19 & 5.80 & 0.12 & 0.33 & 3.23 \\
\hline 13 & Laurel/ Cordia alliodora & 178 & 65.19 & 0.66 & 1.87 & 18.12 \\
\hline 14 & Madero Negro/ Gliricidia sepium & 8 & 1.39 & 0.02 & 0.06 & 0.57 \\
\hline 15 & Popa/ Hondroanthus chrysanthus & 4 & 1.51 & 0.02 & 0.06 & 0.61 \\
\hline & Total, general & & 334.15 & 3.64 & 10.35 & 100.00 \\
\hline
\end{tabular}


La biomasa promedio encontrada en este estudio $3.64 \mathrm{t} / \mathrm{ha}$, son similares a los rangos encontradas por Ortiz, Riascos y Somarriba (2008), sobre almacenamiento y fijacion de carbono en SAF, quienes determinaron que un SAF con árbol de laurel puede acumular en promedio $1.86 \mathrm{t}$ de biomasa en condiciones de valle y $3.97 \mathrm{t}$ en loma en 25 años. La diferencia puede deberse a la edad de los sistemas puesto que el SAF de Los Laureles se desconoce las edades de las diferentes especies, las forestales y frutales que son las de sombra ya estaban establecidas cuando se sembró el cultivo principal y éste se ha ampliado con plantación de áreas nuevas.

Los resultados obtenidos en este estudio son similares a los reportes de toneladas de biomasa por hectárea obtenidos en el departamento de Nariño- Colombia por Acosta y Tupaz (2007), donde el almacenamiento de la biomasa aérea alcanzó rangos de $0.65 \mathrm{t} / \mathrm{ha}$ a $2.5 \mathrm{t} / \mathrm{h}$, tomando en cuenta que esta investigación se realizó en árboles dispersos y el número de árboles influye sobre la producción de biomasa; no obstante, los resultados del presente estudio se encuentran con 3.64 t/ha de biomasa.

Al igual en los SAF de Talamanca Costa Rica, donde se han encontrado valores de $3.56 \mathrm{t} / \mathrm{ha}$ de biomasa (Chacón, 2007). Los resultados de biomasa son similares con los reportados de investigaciones de otros autores en diferentes condiciones, el componente cacao posee mayor cantidad de biomasa, debido a que es el cultivo principal del sistema con densidad poblacional mayor en comparación a las demás especies de sombra (frutales y forestales).

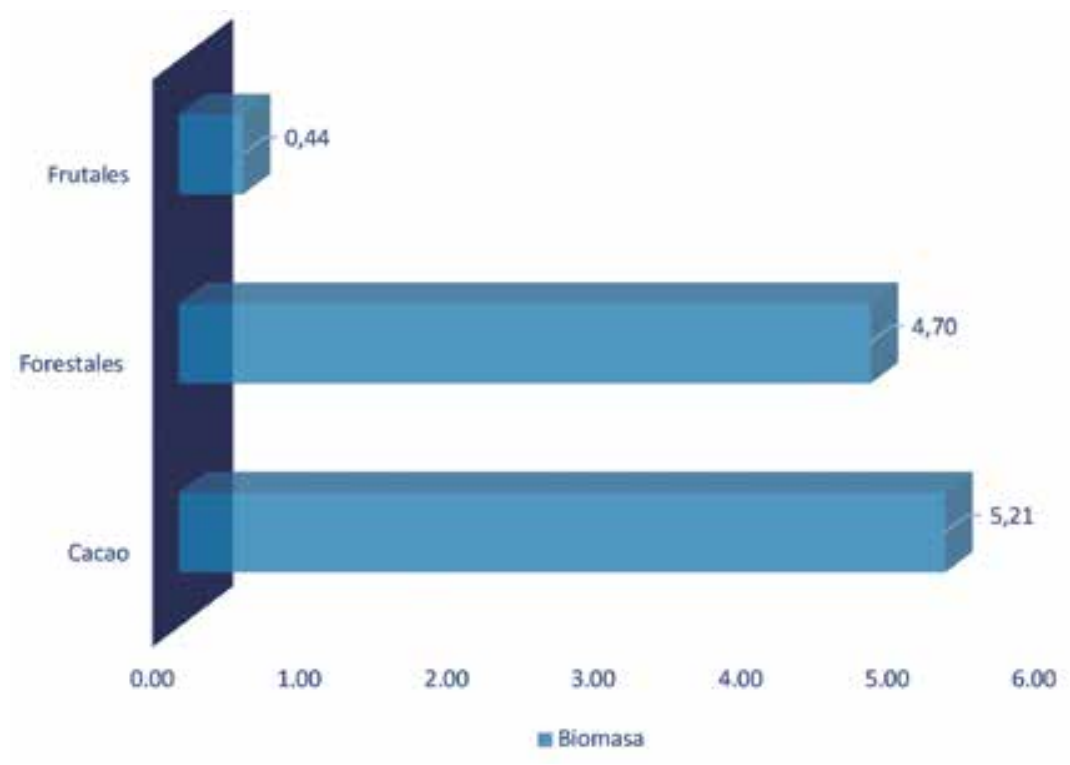

Gráfico 1. Biomasa en árboles de cacao, frutales y forestales.

En el gráfico 1 se muestra los resultados finales del contenido de biomasa del sistema agroforestal con cacao de laboratorio natural Los Laureles, en el cual para la principal especie (cacao) se determinó mediante parcelas de muestreo encontrándose 5.21 toneladas de biomasa y mediante inventarios al 100\% especies frutales 0.44 tonelada y forestales 4.70 toneladas.

Además, la producción de biomasa en los sistemas agroforestales depende de las especies que se encuentra dentro del SAF debido a que el contenido de agua en cada especie es diferente, ya que en el secado de las muestras en esta investigación se observó que el contenido de humedad presenta variaciones de entre las especies, mismo depende de la estructura de la madera como la porosidad, tipo tallo entre otras.

En el cuadro 2, muestra el volumen total y las reservas de carbono por hectárea y toneladas $C$ total del área del sistema agroforestal del laboratorio natural Los Laureles, encontrando mayor contenido de $C$ el 
cacao con $4.17 \mathrm{t}$; laurel $1.50 \mathrm{t}$, aguacate canela con $0.98 \mathrm{t}$, y el resto de las especies almacenaron entre 0.001 a 0.28 toneladas de carbono en biomasa aérea.

Cuadro 2. Volumen y carbono aéreo por especie del SAF en Los Laureles 2018.

\begin{tabular}{|c|c|c|c|c|c|c|c|}
\hline $\mathrm{N}^{\circ}$ & Especie & $\begin{array}{c}\text { N. árboles } \\
\text { total }\end{array}$ & Volumen & t C/ha & $\mathrm{tC}$ & $\mathrm{tC} / \mathrm{m}^{3}$ & $\%$ \\
\hline 1 & Cacao/Theobroma cacao L & 2424 & 208.97 & 1.47 & 4.17 & 0.020 & 50.46 \\
\hline 2 & Aguacate Canela/Persea coerulea & 7 & 23.52 & 0.34 & 0.98 & 0.041 & 11.81 \\
\hline 3 & Cedro Real/ Cedrela Odorata & 61 & 3.96 & 0.03 & 0.09 & 0.024 & 1.14 \\
\hline 4 & $\begin{array}{l}\text { Costilla de Danto/ Lecointea amazo- } \\
\text { nica }\end{array}$ & 1 & 0.34 & 0.01 & 0.02 & 0.063 & 0.26 \\
\hline 5 & Elequeme/ Erythrina poeppigiana & 3 & 8.98 & 0.28 & 0.78 & 0.087 & 9.49 \\
\hline 6 & Guarumo/ Cecropia peltata & 46 & 0.88 & 0.00 & 0.01 & 0.015 & 0.16 \\
\hline 7 & Guácimo molenillo/ Luehea candida & 21 & 5.80 & 0.09 & 0.26 & 0.045 & 3.16 \\
\hline 8 & Laurel/ Cordia alliodora & 8 & 65.19 & 0.53 & 1.50 & 0.023 & 18.16 \\
\hline 9 & Madero Negro/ Gliricidia sepium & 2 & 1.39 & 0.02 & 0.04 & 0.032 & 0.54 \\
\hline 10 & Popa/ Hondroanthus chrysanthus & 37 & 1.51 & 0.02 & 0.05 & 0.033 & 0.61 \\
\hline 11 & Aguacate/ Persea americana & 4 & 2.14 & 0.01 & 0.02 & 0.008 & 0.21 \\
\hline 12 & Guaba/Inga edulis & 19 & 7.42 & 0.10 & 0.28 & 0.038 & 3.41 \\
\hline 13 & Limón/ Citrus aurantifolia & 178 & 0.04 & 0.00 & 0.001 & 0.030 & 0.01 \\
\hline 14 & Mango/ Magnifera indica & 8 & 1.09 & 0.01 & 0.03 & 0.031 & 0.41 \\
\hline \multirow[t]{2}{*}{15} & Pijibay/ Bactris gasipaes & 4 & 2.93 & 0.00 & 0.01 & 0.005 & 0.17 \\
\hline & Total, general & & 334.15 & 2.91 & 8.26 & 0.025 & 100.0 \\
\hline
\end{tabular}

Estudio realizado por Espinoza, Vásquez, y Torres (2012) sobre almacenamiento de carbono en SAFcafé, mencionan que los sistemas agroforestales almacenan aproximadamente $9,21,50$ y $63 \mathrm{t} C$ ha-1, en la biomasa aérea y raíces de regiones semiáridas, subhúmedas, húmedas y templadas, respectivamente y el carbono en todos los almacenes se estima entre 12 y 228 t $C$ ha. Mientras tanto, en el presente estudio sobre Carbono en biomasa aérea de sistema agroforestal con cacao se encontró 2.91 toneladas de carbono por hectárea, la cual es menor en comparación con el estudio de Espinoza, ésta diferencia se debe a que el estudio de los otros autores antes mencionado calcularon el Carbono en sistema radicular, suelo y biomasa aérea del SAF, puesto que del $100 \%$ de carbono almacenado en sistemas agroforestales el $51.9 \%$ en el suelo, $35.9 \%$ está en biomasa aérea, $7.5 \%$ raíces gruesas, $4.3 \%$ raíces delgadas y un $0.3 \%$ en necromasa y hojas según estudio realizado en Waslala- Nicaragua por (Poveda, 2013).

López (2011), realizó un inventario de almacenamiento carbono en sistemas agroforestales de laurel - cacao en Turrialba Costa Rica, en donde menciona que las plantas de laurel dispersos dentro del SAF aporta $0.75 \mathrm{t} \mathrm{C/ha}$ al sistema a los 17 años. Sin embargo, en el sistema agroforestal del laboratorio natural Los Laureles, el laurel aporta o.53 t C/ha, diferencia debida a la edad de los SAF, densidad arbórea de esta especie en cada sistema.

En Caldas, Colombia, Aristizabal y Guerra (2002), se estimó el almacenamiento de carbono en un sistema agroforestal laurel - cacao en un período de 15 años, donde encontró una tasa de almacenamiento de $3.05 \mathrm{t} \mathrm{C}$ /ha, la cual es similar a la tasa de almacenamiento del SAF de Los Laureles en donde estimó $2.91 \mathrm{t}$ $\mathrm{C} / \mathrm{ha}$, la diferencia puede deberse a la edad de los SAF ya que el sistema agroforestal del presente estudio desconoce la edad de las especies de sombra las cuales son forestales y frutales. Por otro lado, influye las especies de sombra en los sistemas las cuales son diferentes de un sistema agroforestales con otro SAF. 
En caso del cacao, los mismos investigadores estimaron que a los 15 años de edad esta especie aporta hasta 1.93 toneladas de carbono por hectárea al sistema agroforestal, combinados con otras especies y con densidad de siembra estable de $4 \mathrm{X}_{3} \mathrm{~m}$. Resultados que supera a la tonelada de carbono almacenado en cacao del SAF de Los Laureles (1.47 t C / ha). La diferencia se debe a la densidad de siembra que tiene los dos estudios ya que el sistema agroforestal del presente estudio tiene una distancia de siembra de $4 \times 4 \mathrm{~m}$, la cual tiene variación en número de plantas e igual, la edad, las condiciones edafoclimáticas, topografía entre otros, los cuales influye en el almacenamiento de carbono.

Se encontró 1.93 toneladas de carbono por hectárea en los sistemas agroforestales de Valle del CieloGuatemala, investigación realizada por Cerda (2011), sobre almacenamiento de carbono en los SAF de 7 años. Lo cual es menor el contenido de carbono en comparación los reportados en esta investigación. Por otro lado, según el mismo autor, en su proyecto "Almacenamiento de carbono en sistemas agroforestales de cacao de Centroamérica", la mayor acumulación de carbono se da hasta los primeros 10 años de edad. En cambio, de acuerdo con Aristizabal y Guerra (2002), hasta los 15 años hay una alta acumulación de carbono (7 t C/ha).

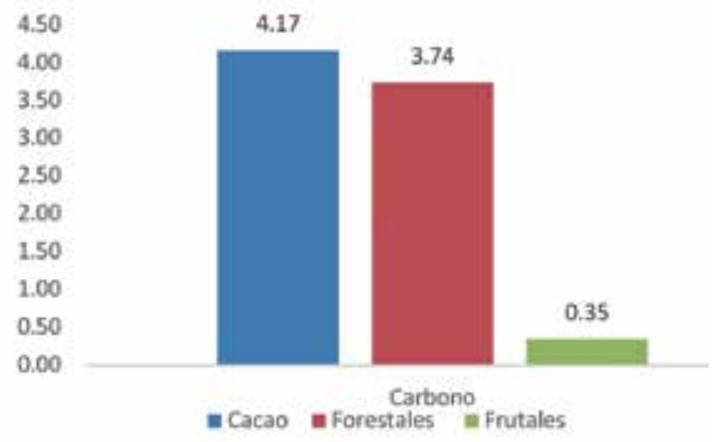

Gráfico 2. Carbono en cacao, frutales y forestales del SAF Los Laureles 2018.

En el gráfico 2 se muestra los resultados del contenido de carbono del sistema agroforestal del laboratorio natural Los laureles que tiene una combinación de cacao como cultivo principal y árboles frutales y especies forestales como sombra permanente.

El almacenamiento de carbono en el sistema agroforestal Los Laureles 2018, depende directamente de la producción de biomasa seca, densidad arbórea y de las especies. Por lo que las especies que poseen mayor cantidad de biomasa a nivel del área del sistema agroforestal resultaron con mayor producción de carbono.

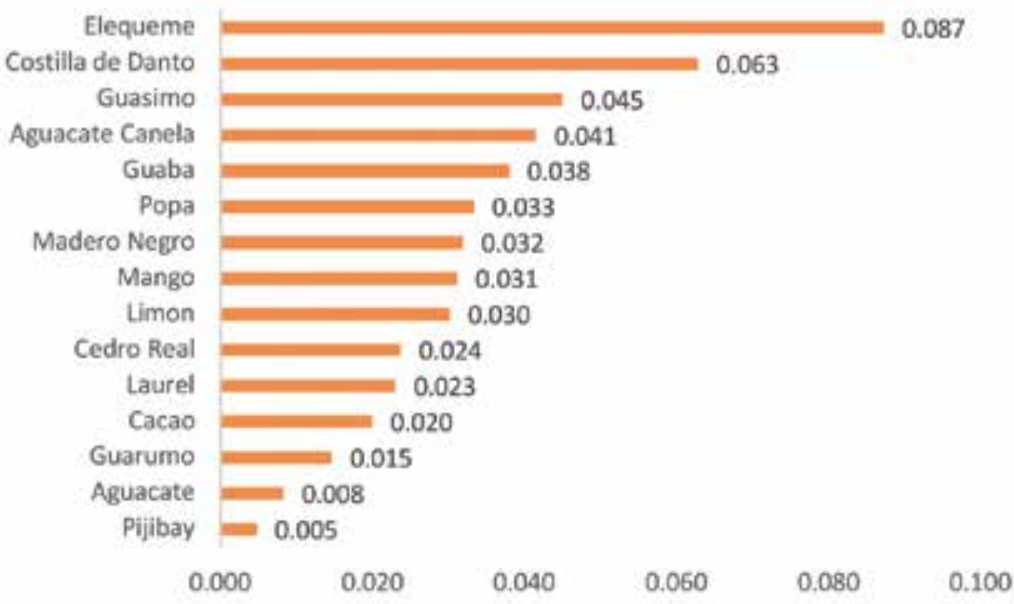

Gráfico 3. Carbono por m³ de las especies del SAF de Los Laureles 2018. 
El almacenamiento de carbono depende directamente del estado, estructura, textura y composición de cada especie. Por lo tanto, las especies que resultaron con mayor contenido de carbono por $\mathrm{m}^{3}$ fueron: elequeme (Erythrina poeppigiana), costilla de danto (Lecointea amazónica), y guásimo (Luehea candida).

Sin embargo, en los datos que se muestran en el cuadro 6 las especies que presentan mayor cantidad de carbono son: cacao (Theobroma cacao L), laurel (Cordia alliodora) y aguacate canelo (Persea coerulea), pero esto se debe a la cantidad de árboles que hay en el sistema, no a que son las que tienen la mayor capacidad de almacenamiento. Una vez estimado el carbono almacenado en un SAF, conceptualmente el proceso de valoración económica del servicio ambiental es relativamente fácil.

En el cuadro 3, muestra que, en la práctica la valoración se complica por el hecho de que todavía no existe un mercado abierto y estable para el secuestro de carbono en el país. Por consiguiente, el SAF del laboratorio natural Los Laureles contiene $2.91 \mathrm{t} \mathrm{C/ha}$ equivalente a 10.6727 t CO2/ha. Tomando como referencia al precio del stock actual de carbono en los mercados voluntarios estaría valorando \$53.36/ha.

\begin{tabular}{|} 
Cuadro 3. Valoración económica ambie \\
\begin{tabular}{|c|c|c|}
\hline Variables & Toneladas & Pago por stock (\$) \\
\hline Carbono aéreo total & 8.26 & \\
\hline $\mathrm{CO}_{2}$ total & 30.31 & 151.55 \\
\hline Carbono aéreo /ha & 2.91 & \\
\hline $\mathrm{CO}_{2} / \mathrm{ha}$ & 10.67 & 53.36 \\
\hline
\end{tabular}
\end{tabular}

Poveda (2013), usó el mismo método y valoró teóricamente el pago anual que un productor percibiría por el servicio ambiental de secuestro de carbono aéreo. Quien tomó como referencia el precio modal pagado en los mercados voluntarios de carbono durante últimos 7 años ( $\$ 5 \mathrm{t} C$ ). Encontrándose $5.4 \mathrm{t} C \mathrm{ha} /$ año, equivalentes a 19.8 t CO2 ha año; por consiguiente, cada productor podría percibir $\$ 99$.

A partir de estos resultados conocemos más a fondo la importancia que radican los árboles en asocio con cacao aparte de su producción como cultivo es un banco de almacenamiento natural del dióxido de carbono el cual contribuye a disminuir las concentraciones de $\mathrm{CO}_{2}$ en la atmósfera, fenómeno conocido como fijación o secuestro de carbono. Sin embargo, la mayor parte de la población de los productores de cacao desconoce la importancia socio ambiental de los sistemas agroforestales que juegan un papel clave en la disminución de las concentraciones de $\mathrm{CO}_{2}$ en la atmósfera y adaptación de los efectos negativos del cambio climático.

\section{Conclusiones}

Los resultados de cuantificación de biomasa aérea del sistema agroforestal del laboratorio natural Los Laureles, obtuvimos: frutales $0.44 \mathrm{t}$, forestales $4.70 \mathrm{t}$ y el cacao con $5.21 \mathrm{t}$ de biomasa. El sistema agroforestal como banco de almacenamiento de Carbono posee 4.09 t $C$ acumuladas en la biomasa aérea de las especies forestales y frutales; por otro lado, la especie de cacao en su biomasa aérea tiene 4.17 t $C$, es así que el SAF logra acumular 8.26 C.

La valoración económica ambiental del sistema agroforestal de cacao con especies frutales y forestales, del laboratorio natural Los Laureles. Corresponde a \$53,36 por hectárea.

\section{Agradecimiento}

Esta publicación obtuvo el financiamiento de: El Fondo de Asistencia Internacional de los Estudiantes y Académicos Noruegos (SAIH). 


\section{Lista de referencias}

Acosta , J. J., \& Florez, F. (Septiembre de 2007). CATIE. Obtenido de http://orton.catie.ac.cr/repdoc/ A11212e/A11212e.pdf

Acosta, \& Tupaz. (2007). Cuantificación de captura de carbono por la biomasa aérea en dos arreglos agroforestales de la Granja Experimental Botana. Nariño, Colombia.

Aristizabal, J., \& Guerra, A. (2002). Estimación de la tasa de Fijación de Carbono en el Sistema Agroforestal nogal Cafetero Cordia Alliodora - Theobroma cacao - Musa paradisiaca. Bogota, Colombia.

Casanova L, F. (o5 de Nobiembre de 2010). Revista Chapingo serie ciencias forestales y del ambiente. Obtenido de http://www.scielo.org.mx/scielo.php?script=sci_arttext\&pid=S2007-40182011000100013

Cerda, R. (2011). Almacenamiento de carbono en sistemas agroforestales de cacao de Centroamérica. Panamá.

Chacón, M. (2007). Almacenamiento de carbono en el suelo, la biomasa arbórea eb sistemas de usos de la tierra en paisajes ganaderos. Bogota, Colombia.

Espinoza, W., Vasquez, A., \& Torres, A. (2012). Almacén de Carbono en Sistemas Agroforestales con Café. Chapingo, México. doi:http://www.scielo.org.mx/pdf/rcscfa/v18n1/v18n1a6. pdf\#page $=13 \&$ zoom $=150,33,7$

López, A. (2011). Aporte de los Sistemas Agroforestales al secuestro de Carbono. Turrialba, Costa Rica.

Ortiz, Á., Riascos, L., \& Somarriba, E. (2008). SIDALC. Obtenido de http://www.sidalc.net/es/sobresidalc

Poveda, V. (22 de 7 de 2013). Repositorio Institucional CATIE. Recuperado el 18 de Enero de 2018, de http:// repositorio.bibliotecaorton.catie.ac.cr/handle/11554/7937 